\title{
Evaluation of Resistance to Ciprofloxacin and Identification of Mutations in Topoisomerase Genes in Escherichia coli and Klebsiella pneumonia Isolated from Pediatric Urinary Tract Infections
}

\author{
(1) Keyghobad Ghadiri1, (1) Alisha Akya1, (1) Azam Elahi², (1) Somaye Jafari1, (1) Roya Chegenelorestani1 \\ ${ }^{1}$ Kermanshah University Medical Sciences, Infectious Diseases Research Center, Kermanshah, Iran \\ 2Emam Reza Hospital, Kermanshah University Medical Sciences, Kermanshah, Iran
}

\begin{abstract}
Aim: Urinary tract infection (UTI) is one of the common infectious diseases of children. Due to the limited use of fluoroquinolones in children, they still have no resistance problems as seen in the adult population. However, recent reports suggested an increase in resistance to fluoroquinolones among bacteria causing UTI in children. Therefore, the aim of this study is to evaluate the prevalence of Escherichia coli and Klebsiella pneumoniae isolates resistant to ciprofloxacin and to detect mutations in their gyrA and parC genes.

Materials and Methods: The present study is conducted on 78 bacterial strains isolated from children with UTI during $2016-2017$ at Imam Reza Hospital in Kermanshah, Iran. The bacteria were identified based on microbiological methods and an antibiotic susceptibility test using disc diffusion and broth microdilution methods. Then, polymerase chain reaction and sequencing were performed to investigate mutations in the gyrA and parC genes.

Results: Overall, $15.3 \%$ of isolates of $E$. coli and K. pneumonia were resistant to ciprofloxacin. Sequence analysis confirmed mutations in the gyrA and parC genes in all of the isolates resistant to ciprofloxacin. The results showed changes in amino acids (ser83leu, ser83phe and Asp87Asn) in codons 83 and 87 in the quinolone resistance-determining regions of the gyrA gene, three substitutions in both the 80 and 84 positions in the parC, ser80lle, Glu84val and Glu84lys genes.

Conclusion: The results of this study revealed resistance to ciprofloxacin in the pediatric population. Given that the use of ciprofloxacin in children is limited, this resistance cannot be due to antibiotic selective pressure. On the other hand, the mutations in the gyrA and parC genes in children was similar to that in adults which indicate that these resistant isolates can be transmitted from adults to children.

Keywords: Urinary tract infections, ciprofloxacin, resistance, fluoroquinolone, Escherichia coli, Klebsiella pneumonia
\end{abstract}

\section{Introduction}

The urinary tract infections (UTIS) are some of the most important diseases among children. The common UTI pathogens among children are the bacteria in the
Enterobacteriaceae family, such as Escherichia coli and Klebsiella pneumonia (1). Despite the fact that beta-lactam antibiotics, cotrimoxazole and ampicillin are the first line of medicine for the experimental treatment of patients with UTI, there are reports of high resistance to these antibiotics 
(2). It is important to select an effective antibiotic in experimental therapy because of the high susceptibility rate, complications and the imposition of treatment costs in pediatric UTIs (3). Although fluoroquinolones are unsuitable for people under the age of 18 , due to increased resistance to cephalosporin, these antibiotics can be used to treat of UTIs caused by E. coli and multidrug-resistant Gram-negative bacteria in patients aged 1-17 years (4). Although the use of these antibiotics is limited in children, fluroquinolone resistant strains are abundant. According to previous reports, the resistance to ciprofloxacin in strains of $E$. coli isolated from children with UTI has increased from $1 \%$ to $10 \%$ and $0.6 \%$ to $4 \%$ between 2002 and 2009 (5). E. coli and K. pneumonia are of the most important fluoroquinolones resistant pathogens (6). The topoisomerase II and IV enzymes are involved in bacterial genome replication, and are the main target of fluoroquinolones. The fluoroquinolones, by inhibiting the activity of these enzymes, inhibit the synthesis of bacterial DNA (7). The DNA gyrase consists of two subunits that are coded as gyrA and gyrB. The topoisomerase IV and consists of two subunits encoded by par $C$ and parE genes. Mechanisms of resistance to quinolones include; 1 ) mutation in the quinolone resistance-determining region (QRDR) of DNA gyrase and topoisomerase IV, 2) intracellular reduction of the drug due to increased expression of efflux pumps or enhanced cell wall impermeability, and 3) production of plasmid-mediated quinolone resistance genes. The main mechanism of resistance is due to mutations in the QRDR region of DNA gyrase and topoisomerase IV. The common location for mutation in E. coli and K. pneumonia is the gyrA gene. Most mutations have been detected in the limited region of QRDR that codes the amino acids 67 to 106 . The most common mutations in the gyrA gene occur in the nucleotides 248 and 260, which cause changes in the amino acids of ser83 and Asp87; and the most common mutations in the parC gene are in the nucleotides 238 and 250 , which cause changes in the amino acids of ser 80 and Glu84 (8-10). In position 83 of the gyrA gene, the amino acid serine is usually replaced by leucine, followed by ser83val and ser83Ala; these alterations increase the Minimum Inhibitory Concentrations (MIC) value of ciprofloxacin. The higher MIC value for ciprofloxacin usually occurs due to mutations in ser83 and Asp87. The frequency of mutations in the QRDR region of gyrA and parC is more common than gyrB and parE. In addition, there is a high level of resistance to fluoroquinolones in isolates with mutations in the QRDR region of parC due to a mutation in gyrA, but the mutations in gyrB and parE have only a complementary role for resistance $(7,9)$. Concerning the resistance to fluoroquinolones, the population of children has not yet encountered the challenges of resistance found in adult populations, but it is important to assess the resistance to fluoroquinolones in children; therefore, this study aimed to evaluate $E$. coli and $K$. pneumonia isolates as the ciprofloxacin-resistant UTI pathogens in children and to detect the mutations in the gyrA and parC genes and their association with MIC for ciprofloxacin.

\section{Materials and Methods}

\section{Bacterial Isolates}

This study was performed on all urine specimens of children under 18 years of age who were referred to the İmam Reza Hospital in Kermanshah between 2016 and 2017. The cases were community acquired UTIs. Exclusion criteria were an age of over 17 years, negative urine culture, patients with a colony count less than $10^{5}(11)$. The urine samples were collected by midstream or urine bags. Following this, bacteriological and biochemical tests were used to detect bacteria in all urine specimens (12).

The study was approved by the Kermanshah University Ethics Committee (approval number: 2016/241). All patients were hospitalized in an university hospital and a free and informed consent was obtained from each participatnts.

\section{Antibiotic Susceptibility Testing}

The susceptibility of isolates to Ciprofloxacin $(5 \mu \mathrm{g})$, Imipenem (10 $\mu \mathrm{g})$, Ampicillin $(10 \mu \mathrm{g})$, Aztreonam (30 $\mu \mathrm{g})$, Ceftazidime $(30 \mu \mathrm{g})$, Cefotaxime $(30 \mu \mathrm{g})$, Ceftriaxone $(20 \mu \mathrm{g})$, Gentamicin $(30 \mu \mathrm{g})$, Tobramycin $(10 \mu \mathrm{g})$ and Cotrimoxazole $(25 \mu \mathrm{g})$ (MAST, England) was conducted using a disk diffusion test according to the Clinical and Laboratory Standards Institute (CLSI) guidelines (13). Determination of MIC of Ciprofloxacin (Sigma, USA) was performed by the broth microdilution method according to CLSI criteria (13). The E. coli ATCC 25922 strain was used as the control strain. The CLSI breakpoints were used for ciprofloxacin susceptibility (susceptible $\leq 1 \mu \mathrm{g} / \mathrm{mL}$; resistant $\geq 4 \mu \mathrm{g} / \mathrm{mL}$ ).

\section{PCR Amplification and Sequencing}

Bacterial DNA was extracted using a genomic DNA purification kit (SinaClon, Iran). The QRDR of the parC and gyrA genes from susceptible and resistant isolates was amplified by PCR using the specific oligonucleotide primers listed in Table I $(14,15)$. The PCR products were detected on $1 \%$ agarose gel after electrophoresis, the DNA bands were visualized by GelDoc apparatus (BioRad, USA). All 
PCR products for the parC and gyrA genes were purified with a PCR purification kit and sequenced (SinaColon, Iran). Sequence data were analyzed for homology with genetic data using the National Center for Biotechnology Information GenBank database (http:/www.ncbi.nlm.nih. gov/BLAST/).

\section{Statistical Analysis}

All data were analyzed using statistical methods and SPSS version 20. The correlation between mutations and the ciprofloxacin MIC was investigated by Sperman's, MannWhitney and Kruskal-Wallis tests. The chi-square test was used to compare resistance of ESBL producing and nonproducing isolates. Statistical significance was defined as having a $p$ value less than 0.05 .

\section{Results}

In this study, 66 isolates of $E$. coli and 12 isolates of $K$. pneumonia of children aged under 18 years were evaluated. The number of girls and boys was 55 (70.5\%) and 23 (29.5\%), respectively. The age distribution among the 66 $(84.6 \%)$ patients with $E$. coli infections was as follows; 34 $(51.5 \%)$ were in the age group of $1-6$ years, 8 (12.1\%) patients were between 7 and 10 years, $9(13.6 \%)$ patients were in the 11-14 years group while the remaining 15 (22.7\%) patients were between 15 and 17 years. The age distribution of the
$12(15.4 \%)$ patients with $K$. pneumonia infections was as follows; 10 (83.3\%) patients were $1-6$ years old, 1 (8.3\%) patient was between 7 and 10 years old and 1 (8.3\%) was between 15 and 17 years old. The mean age of patients was $6.1 \pm 5.59$ (maximum of 17 years and minimum of 1 year).

The antibiotic susceptibility pattern to 10 antibiotics and MIC for ciprofloxacin in the 66 isolates of E. coli and 12 isolates of $K$. pneumoniae are presented in Table II and Figure 1. The highest antibiotic resistance was observed for ampicillin and cotrimoxazole. E. coli strains showed the lowest resistance to Gentamicin, ciprofloxacin and aztreonam while $K$. pneumonia isolates exhibited the least resistance to Tobramycin and ciprofloxacin. No resistance to imipenem was found in either bacteria studied.

Of the 78 isolates, 18 (23.07\%) were ESBL producers. Of these 18 isolates, 6 (33.3\%) were resistant to ciprofloxacin. Resistance to ciprofloxacin in ESBL-producing isolates was higher than isolates without ESBL ( $p=0.008)$ (Table III).

The nucleotide sequence of the QRDR region from gyrA and parC indicated the presence of two mutations in gyrA and two mutations in parC.

DNA sequence analysis of the QRDR of gyrA showed that all isolates of $E$. coli and $K$. pneumonia resistant to ciprofloxacin showed mutations in gyrA at codon 83 and codon 87.

Table I. The primers

\begin{tabular}{|l|l|l|l|l|}
\hline Gene & Primer & Target site & Amplicon size (bp) & Reference \\
\hline $\begin{array}{l}\text { parC F } \\
\text { parC R }\end{array}$ & $\begin{array}{l}\text { 5'AGCGCCTTCCGTACATGAAT3' } \\
\text { 5'GTGGTAGCGAAGAGGTCGTT3' }\end{array}$ & QRDR of parC & 964 & $(14)$ \\
\hline $\begin{array}{l}\text { gyrA F } \\
\text { gyrA R }\end{array}$ & $\begin{array}{l}\text { 5'TACACCGGTCAACATTGAGG3' } \\
\text { 5'CCGGATCGGTAAGCTTCTTCAAT3' }\end{array}$ & QRDR of gyrA & 684 & $(15)$ \\
\hline
\end{tabular}

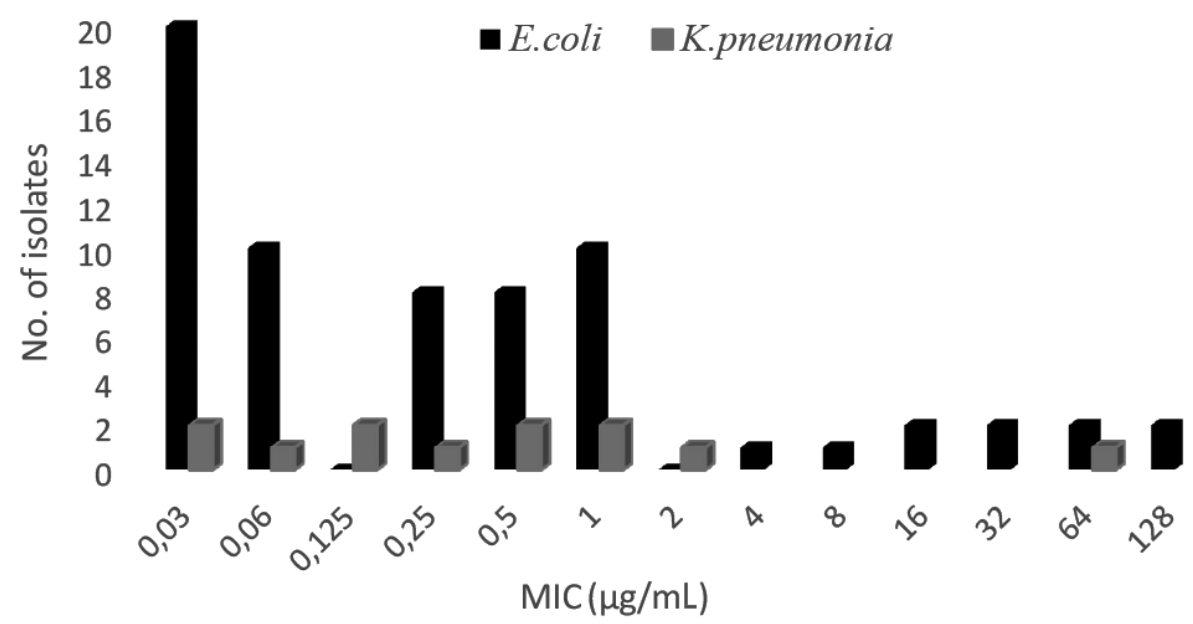

Figure 1. The distribution of isolates according to their Minimum Inhibitory Concentrations level $(\mu \mathrm{g} / \mathrm{mL})$ for ciprofloxacin 
The results showed that mutations mapped in the parC gene conferring resistance to iprofloxacin were either in the codon Ser80 to llu80 or in codon Clut84 to Val 84 for E. coli (Table IV). Whereas the K. pneumoniae parC mutant conferring resistance to ciprofloxacin was Glut84 to Lysine84 (Table IV).

As shown in Table III, the Ser83 $\rightarrow$ Leu + Asp87 $\rightarrow$ Asn mutation in the gyrA gene and Ser $80 \rightarrow$ ILe mutation in the parC gene were the most frequent types in those isolates resistant to ciprofloxacin. Curiously, one of the ciprofloxacin sensitive isolate of $E$. coli exhibited a mutation in the gyrA gene, but the rest of ciprofloxacin-sensitive $E$. coli and $K$. pneumonia had no mutation in the gyrA and parC genes (Table V).

The MIC value of ciprofloxacin was higher in those isolates with multiple mutations in the gyrA and parC genes compared to isolates with a single mutation in the gyrA gene or without any mutations in the gyrA and par $C$ genes $(p=0.001)$.

The nucleotide sequence data of gyrA and parC have been deposited into the GenBank under the accession number of MH425518, MH425519, MH324489, MH324490 and $\mathrm{MH} 523403$.

\section{Discussion}

In this study, the highest antibiotic resistance was observed for ampicillin and cotrimoxazole. Studies in İran have also reported resistance to ampicillin from 88 to 94\% and resistance to cotrimoxazole from 63 to $71 \%$ for E. coli isolates in pediatric UTIs $(2,16,17)$. It seems that the extensive use of ampicillin and cotrimoxazole as empirical therapy for UTI has resulted in the high resistance of $E$. coli isolates to these antibiotics in İran $(18,19)$. In this and other studies, resistance to imipenem has not been observed in $E$. coli causing UTI; therefore, this drug is still an effective one in the treatment of UTI (18).

In the present study, the prevalence of UTI was higher in girls than in boys, which is consistent with other studies $(2,18)$, due to the structure and anatomy of the female urogenital system (18). Since fluoroquinolones are less commonly used in children, they have not yet encountered the resistance problems occurring in adults (20). Our

Table II. Antibiotic susceptibility of Escherichia coli and Klebsiella pneumonia isolated from children Urinary tract infections

\begin{tabular}{|c|c|c|c|c|c|c|}
\hline \multirow[b]{2}{*}{ Antimicrobial agent } & \multicolumn{3}{|c|}{ Escherichia coli (66) } & \multicolumn{3}{|c|}{ Klebsiella pneumonia (12) } \\
\hline & $\mathbf{R}$ & $\mathbf{I}$ & $S$ & $\mathbf{R}$ & I & $\mathbf{S}$ \\
\hline Imipenem & $0(0)$ & $0(0)$ & $66(100)$ & $0(0)$ & & $100(12)$ \\
\hline Ampicillin & $74.2(49)$ & $6.1(4)$ & $19.7(13)$ & $100(12)$ & $0(0)$ & $0(0)$ \\
\hline Aztreonam & $15.1(10)$ & $3.1(2)$ & $81.8(54)$ & $16.6(2)$ & $0(0)$ & $83.4(10)$ \\
\hline Ceftazidime & $21.2(14)$ & $3.1(2)$ & $75.7(50)$ & $25(3)$ & $0(0)$ & $75(9)$ \\
\hline Cefotaxime & $25.7(17)$ & $4.5(3)$ & $69.7(46)$ & $16.7(2)$ & $8.3(1)$ & $75(9)$ \\
\hline Ceftriaxone & $22.7(10)$ & $0(0)$ & $77.3(51)$ & $16.7(2)$ & $8.3(1)$ & $75(9)$ \\
\hline Gentamicin & $15.1(10)$ & $1.5(1)$ & $83.3(55)$ & $0(0)$ & $0(0)$ & $100(12)$ \\
\hline Tobramycin & $18.2(12)$ & $6.1(4)$ & $75.7(50)$ & $8.3(1)$ & $8.3(1)$ & $83.4(10)$ \\
\hline Ciprofloxacin & $15.1(10)$ & $0(0)$ & $84.8(56)$ & $8.3(1)$ & $0(0)$ & $91.7(11)$ \\
\hline Cotrimoxazole & $40.9(27)$ & $4.5(3)$ & $54.5(36)$ & $33.3(4)$ & $0(0)$ & $66.7(8)$ \\
\hline
\end{tabular}

R: Resistance, I: Intermediate, S: Susceptible

Table III. Ciprofloxacin susceptibility of ESBL-producing and non-ESBL producing Escherichia coli isolates

\begin{tabular}{|l|l|l|l|}
\hline \multirow{2}{*}{ Isolates } & \multicolumn{3}{l}{ Frequency of ciprofloxacin susceptibility (no.) } \\
\cline { 3 - 4 } & Resistant & Sensitive \\
\hline \multirow{2}{*}{ Klebsiella pneumonia (12) } & ESBL-producing isolates & 0 & 2 \\
\cline { 2 - 5 } & Non ESBL-producing isolates & 1 & 9 \\
\hline \multirow{2}{*}{ Escherichia coli (66) } & ESBL-producing isolates & 6 & 10 \\
\cline { 2 - 4 } & Non ESBL-producing isolates & 4 & 46 \\
\hline
\end{tabular}

ESBL: Extended-Spectrum Beta-Lactamase 
findings showed that ciprofloxacin-resistant isolates can also be found in children. A study in Yasuj, Iran, reported an increase in the rate of resistance to ciprofloxacin in children (19). In a study by Domiınguez et al. (21), 5\% of E. coli strains isolated from children were resistant to ciprofloxacin. Other studies in recent years have also documented isolates of quinolone-resistant Enterobacteriaceae in children $(22,23)$. Reports from İran and other parts of the world demonstrated a significant correlation between the mutations in the chromosomal gyrA and parC genes and resistance to fluoroquinolones $(24,25)$. In the present study, mutations were observed in the gyrA and parC genes among all isolates resistant to ciprofloxacin, and sensitive isolates also lacked mutation in these genes. Further, the average MIC level of fluoroquinolones was higher in those isolates with mutations in comparison to those isolates without mutations $(p=0.001)$, which highlights the important role of mutations in resistance.

Recently, in a report from Spain, a mutation in the gyrA gene was found in isolates from infants, which play a role in resistance to quinolones (26). In our research, similar to a study by Huang et al. (6), the results of the sequencing of QRDRs from gyrA showed the presence of Ser83 $\rightarrow$ Leu + Asp87 $\rightarrow$ Asn mutations among quinolone-resistant isolates from children, as the most frequent mutations. In this study, the mutations in the

Table IV. gyrA and parC mutations in Escherichia coli and Klebsiella pneumonia isolates

\begin{tabular}{|c|c|c|c|c|c|}
\hline & Gene & Amino acid position & $\begin{array}{l}\text { Nucleotide } \\
\text { changes }\end{array}$ & $\begin{array}{l}\text { Amino acids } \\
\text { substitute }\end{array}$ & No. of isolates (\%) \\
\hline \multirow{7}{*}{ gyrA } & \multirow{4}{*}{ Escherichia coli } & \multirow{2}{*}{ Serine83/Asp87 } & TCG $\rightarrow$ TTG & Leucine & \multirow{2}{*}{$10(15.1)$} \\
\hline & & & $\mathrm{GAC} \rightarrow \mathrm{AAC}$ & Asparagine & \\
\hline & & Serine83 & TCG $\rightarrow$ TTG & - & $1(1.5)$ \\
\hline & & WT & - & - & $55(83.3)$ \\
\hline & \multirow{3}{*}{ Klebsiella pneumonia } & 7.5 & $\mathrm{TCC} \rightarrow \mathrm{TTC}$ & Phenylalanine & \multirow[t]{2}{*}{$1(8.3)$} \\
\hline & & SЕIILOSJASPOI & $\mathrm{GAC} \rightarrow \mathrm{AAC}$ & Asparagine & \\
\hline & & WT & - & - & $11(91.7)$ \\
\hline \multirow{6}{*}{ parC } & \multirow{4}{*}{ Escherichia coli } & & $\mathrm{AGC} \rightarrow \mathrm{ATT}$ & Isoleucine & \multirow{2}{*}{$3(4.5)$} \\
\hline & & 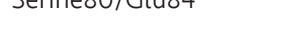 & $\mathrm{GAC} \rightarrow \mathrm{GTA}$ & Valin & \\
\hline & & Serine 80 & $\mathrm{AGC} \rightarrow \mathrm{ATT}$ & Isoleucine & $7(10.6)$ \\
\hline & & WT & - & - & $56(84.8)$ \\
\hline & \multirow{2}{*}{ Klebsiella pneumonia } & Glu84 & $\mathrm{GAA} \rightarrow \mathrm{AAA}$ & Lysine & $1(8.3)$ \\
\hline & & WT & - & - & 11 (91.7) \\
\hline
\end{tabular}

Asp: Aspartic acid, Glu: Glutamic acid

Table V. Mutations in Quinolone resistance-determining regions of the gyrA and parC genes of Escherichia coli and Klebsiella pneumonia in isolates and their corresponding Minimum Inhibitory Concentrations for Ciprofloxacin

\begin{tabular}{|c|c|c|c|c|c|c|c|c|c|c|}
\hline & \multicolumn{4}{|c|}{ Mutations in the QRDR } & \multirow{3}{*}{ No. of isolates } & \multirow{2}{*}{\multicolumn{5}{|c|}{ MIC $(\mu \mathrm{g} / \mathrm{mL})$ range }} \\
\hline \multirow{6}{*}{ E. coli } & \multicolumn{2}{|l|}{ gyrA } & \multicolumn{2}{|l|}{ parC } & & & & & & \\
\hline & Ser83 & Asp87 & Ser80 & Glu84 & & $<1$ & $1-2$ & $4-8$ & $16-32$ & $64-128$ \\
\hline & Leu & Asn & Ile & Val & 3 & - & - & - & - & 3 \\
\hline & Leu & Asn & Ile & - & 7 & - & - & 2 & 4 & 1 \\
\hline & Leu & - & - & - & 1 & - & 1 & - & - & - \\
\hline & - & - & - & - & 55 & 46 & 9 & - & - & - \\
\hline$K$ nnoumsni & Phe & Asn & - & Lys & 1 & - & - & - & - & 1 \\
\hline 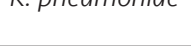 & - & - & - & - & 11 & 8 & 3 & - & - & - \\
\hline
\end{tabular}

Ser: Serine, Ile: Isoleucine; Leu: Leucine, Asp: Aspartic acid, Glu: Glutamic acid, Phe: Phenylalanine, Asn: Asparagine, Val: Valin, Lys: Lysine, QRDR: Quinolone resistancedetermining region, MIC: Minimum Inhibitory Concentrations 
gyrA and parc genes were similar to mutations in these genes of strains isolated from the adult population in our previous study (27). Another study also reported that the Ser83 $\rightarrow$ Leu + Asp87 $\rightarrow$ Asn mutation was similar to that of quinolone-resistant isolates from children and adults (6). In fact, it has been reported that resistant isolates might be transmitted from adults to children (6). The topoisomerase IV is a secondary target in the Gram-negative bacteria for fluoroquinolones (7). In those isolates with mutations in the QRDR region of the parC gene, the level of resistance to ciprofloxacin was higher, which is consistent with other studies that reported that the mutation in the topoisomerase IV reduces the sensitivity to quinolones (28).

In our study, the isolates with multiple mutations in the gyrA and parC genes showed that the MIC value of ciprofloxacin was higher compared to isolates with single or no mutation. These results indicate that multiple mutations are required in these genes to induce high levels of resistance to fluoroquinolones. Faghri et al. (28) reported that it is necessary to have multiple mutations in the gyrA and parC genes for high levels of resistance to fluoroquinolones.

\section{Conclusion}

Resistance to ciprofloxacin is high in E. coli isolated from the pediatric population in Iran. Given that the use of ciprofloxacin in pediatric UTIs is limited, the presence of this fluoroquinolone resistance alone cannot be due to antibiotic selective pressure. At the same time, the mutations in the gyrA and parC genes in E. coli isolated from children were similar to those of adults, indicating the possibility of the transference of these resistant isolates from adults to children.

\section{Acknowledgements}

We would like to thank the Clinical Development Research Unit of Imam Reza Hospital msd the Kermanshah University of Medical Sciences Pulmonary Diseases Unit.

\section{Ethics}

Ethics Committee Approval: The study was approved by the Kermanshah University Ethics Committee (approval number: 2016/241).

Informed Consent: All patients were hospitalized in an university hospital and a free and informed consent was obtained from each participatnts.

Peer-review: Externally and internally peer-reviewed.

\section{Authorship Contributions}

Design: K.G., A.A., A.E., S.J., R.C., Data Collecting or Processing: K.G., A.A., A.E., S.J., R.C., Analysis or Interpretation: K.G., A.A., A.E., S.J., R.C., Literature Search: K.G., A.A., A.E., S.J., R.C., Writing: K.G., A.A., A.E., S.J., R.C.

Conflict of Interest: The authors declare that there was no conflict of interest to publish this article.

Financial Disclosure: Research reported in this publication was supported by Kermanshah University of Medical Sciences, Kermanshah, İran

\section{References}

1. Barzan M, Hoseyni-Doust R, Ghalavand Z. Investigation of frequency and antimicrobial pattern of gram-negative bacteria isolated from urine specimens of children with urinary tract infection in Tehran, Iran. Iran / Med Microbiol 2016;9:99-104.

2. Amini F, Vaziri S, Karimpour HA, Hassani S, Mohamadi S, Azizi $M$. The study of frequency and antibiotic resistance pattern of urinary tract infection pathogens in children of Kermanshah in 2015. RJMS 2017;24:20-7.

3. Bader MS, Haeboldt I, Brooks A. Management of complicated urinary tract infection in the era of antimicrobial resistance. Post Grade Med 2010;122:7-15.

4. Choi SH, Kim EY, Kim YJ. Systemic use of fluoroquinolone in children. Korean / Pediatr 2013;56:196-201.

5. Edlin RS, Shapiro DJ, Hersh AL, Copp HL. Antibiotic resistance patterns of outpatient pediatric urinary tract infections. I Urol 2013;190:222-7.

6. Huang Y, Ogutu JO, Gu J, et al. Comparative Analysis of Quinolone Resistance in Clinical Isolates of Klebsiella pneumoniae and Escherichia coli from Chinese Children and Adults. Biomed Res Int 2015;2015:168292.

7. Hooper DC. Emerging mechanisms of fluoroquinolone resistance. Emerg Infect Dis 2001;7:337-41.

8. Bansal S, Tandon V. Contribution of mutations in DNA gyrase and topoisomerase IV genes to ciprofloxacin resistance in Escherichia coli clinical isolates. Int / Antimicrobial Agents 2011;37:253-5.

9. Ruiz J. Mechanisms of resistance to quinolones: target alterations, decreased accumulation and DNA gyrase protection. J Antimicrob Chemother 2003;51:1109-17.

10. Krishnan S, Balasubramanian D, Raju BA, Lakshmi BS. Use of a naturally occurring codon bias for identifying topoisomerase mutations in ciprofloxacin resistant Escherichia coli using PCR and future prospects with other bacterial genera: A pilot study. Adv Biol Chem 2012;2:366-71.

11. Fauci AS, Braunwald E, Kasper DL. Harrison's principles of internal medicine. 17th ed. USA: McGraw-Hill; 2008.

12. Washington C, Stephen A, Janda W. Koneman's color atlas and textbook of diagnostic microbiology. 6th ed. USA: Lippincott williams wilkins; 2006.

13. Clinical and Laboratory Standards Institute. Performance Standards for Antimicrobial Susceptibility Testing. Supplement T-sl, editor. USA: CLSI; 2015. 
14. Lindgren PK, Karlsson A, Hughes D. Mutation rate and evolution of fluoroquinolon resistance in Escherichia coli Isolates from patients with urinary tract infection. Antimicrob Agents Chemother 2003:3222-32.

15. Park $\mathrm{YH}$, Yoo JH, Huh DH, Cho YK, Choi JH, Shin WS. Molecular analysis of fluoroquinolone-resistance in Escherichia coli on the aspect of gyrase and multiple antibiotic resistance (mar) genes. Yonsei Med J 1998;39:534-40.

16. Ghorashi Z, Ghorashi S, Soltani-Ahari H, Nezami N. Demographic features and antibiotic resistance among children hospitalized for urinary tract infection in northwest Iran. Infect Drug Resist 2011;4:171-6.

17. Barari Sawadkouhi R, Sorkhi H, Pournasrollah M, Bijani A. Antibiotic Resistance of Bacteria Causing Urinary Tract Infections in Children Hospitalized in Amirkola Children Hospital during 2010-2011. J Babol Univ Med Sci 2013;15:89-94.

18. Sharif MR, Nouri S. The Frequency and Antibiotic Resistance of Urinary Tract Infection Organisms in Hospitalized Children. Iran J Infect Dis 2014;19:47-51.

19. Asadi Manesh F, Sharifi A, Mohammad Hosini Z, et al. Antibiotic Resistance of Urinary Tract Infection of Children Under 14 Years Admitted To The Pediatric Clinic of Imam Sajjad Hospital, 2012. Armaghane Danesh 2014;19:411-20.

20. Rose L, Coulter MM, Chan S, Hossain J, Di Pentima MC. Trends of fluoroquinolone-resistant Escherichia coli amongst urinary isolates in children: a 10 year surveillance study. I Med Microbiol 2015;64:778-81.

21. Domiınguez $E$, Zarazaga $M$, Saenz $Y$, Brinas $L$, Torres $C$. Mechanisms of antibiotic resistance in Escherichia coli isolate obtained from healthy children in Spain. Microb Drug Resist 2002;8:321-7.

22. Ayatollahi J, Shahcheraghi SH, Akhondi R, Soluti S. Antibiotic Resistance Patterns of Escherichia coli Isolated from Children in Shahid Sadoughi Hospital of Yazd. Iran / Pediatr Hematol Oncol 2013;3:78-82.

23. Garraffo A, Marguet C, Checouryetal A, et al. Urinarytract infections in hospital pediatrics: many previous antibiotherapy and antibiotics resistance, including fluoroquinolones. Med Mal Infect 2014;44:63-8.

24. Heidari F, Pourahmad R, Shareghi B. Expression of ompF gene in E. coli mutants resistant to ciprofloxacin and Tetracycline. J Genetic Novin 2015;10:123-8.

25. Kmet $V$, Kmetova $M$. High level of quinolone resistance in Escherchia coli from healthy chicken broiler. Folia Microb 2010;55:79-82.

26. Pons MJ, Mosquito S, Gomes C, Del Walle L), Ochoa T), Ruiz J. Analysis of quinolone-resistance in commensal and diarrheagenic Escherichia coli isolates from infants in Lima, Peru. Trans R Soc Trop Med Hyg 2014;108:22-8.

27. Chegene Lorestani R, Akya A, Elahi A. The Mutations of Topoisomerase Genes and Their Effect on Resistance to Fluoroquinolones in Extended-Spectrum $\beta$-LactamaseProducing Escherichia coli. Jundishapur / Nat Pharm Prod 2018;13:e57964.

28. Faghri J, Dehbanipour R, Mobasherizadeh S, Maleki N. Study of Antibiotic Resistance Pattern and Mutation in Genes gyrA and parC of Escherichia Coli Causing Urinary Tract Infection. Sci J Hamdan Univ Med Sci 2016;23:118-25. 